\title{
Modelos 3D de ciudades como herramienta para la estimación de la demanda energética
}

Camilo Alexander León Sánchez*

Fecha de recepción: 13 de septiembre de 2013

Fecha de aprobación: 20 de octubre de 2013

Pp. 125 a 161

\section{RESUMEN}

En esta investigación, se presenta una metodología para la estimación de la demanda de energía eléctrica de edificios, empleando modelos de ciudades 3D. La idea de incluir este tipo de información, es que en la actualidad son ricos en datos geométricos y semánticos, los cuales pueden llegar a ser útiles en el pronóstico de demandas de energía. Inicialmente, se presentan los conceptos básicos de sistemas de información geográfica y de energía eléctrica, siguiendo con la selección del método que mejor se ajuste a los requerimientos del proyecto.

Posteriormente, se presentan las herramientas de procesamiento para archivos CityGML y luego, con la metodología para la estimación de la demanda de energía eléctrica, finalizando con la presentación de un diagrama de actividades, que expresa de mejor forma las ideas expuestas. Después, se presentan los resultados en un área de estudio empleada durante la implementación y finalmente, las conclusiones.

\section{Palabras Clave}

Modelos 3D, energía eléctrica, demanda de energía, sistemas de información geográfica.

* MSc. en Geodesy and Geoinformation Science, Technische Universität Berlin. Berlín Alemania. Ingeniero Catastral y Geodesta, Universidad Distrital. Bogotá Colombia. 


\section{D city models as a tool for the estimation of energetic demand}

\section{AbStract}

In this research, a methodology for the estimation of electrical energy demand of buildings using a $3 D$ city model is described. The idea of including all these data justifies the fact that today these models are full of geometric and semantic data, which could be applied in energetic demand forecasts. At the onset, basic concepts of geographic information systems and electrical energy are presented, following with the best selection method required for this kind of research project.

Later, comprehensive tools for processing CityGML files are presented, including the methodology for the estimation of electrical energy demand, which ends with the presentation of a diagram of activities showing the best way to illustrate the resulting ideas. After that, the outcomes of an applied area of study used during its implementation stage are shown. The final part of this research paper is devoted to derived conclusions.

\section{KEY WORDS}

Electrical Energy Demand, 3D City Models, Geographic Information Systems and Citygml. 


\section{Modélisation de centres urbains en 3D: un outil pour l'estimation de la demande énergétique}

\section{RÉSUMÉ}

Cette étude présente une méthodologie pour l'estimation de la demande en énergie électrique des bâtiments à l'aide de modèles de villes 3D. La volonté d'utiliser ce type de données se fonde sur une quantité d'analyses géométriques et sémantiques actuellement disponibles et pouvant être utiles pour la prévision de la demande énergétique. Nous présenterons les concepts de base des systèmes d'information géographique et énergétique en sélectionnant la méthode qui s'adapte le mieux aux exigences du projet. Nous présenterons ensuite des outils d'analyse du programme CityGML utilisant la méthodologie d'estimation de la demande en énergie électrique et exposerons un diagramme des activités reprenant plus clairement les idées formulées. La dernière partie de ce document sera consacrée aux conclusions de l'étude.

\section{Mots-clés}

Demande en Énergie Électrique, Modèles de Centres Urbains 3D, Système d'Information Géographique, CityGML 


\section{Modelos 3D de cidades como ferramenta para estimar a demanda de energia}

\section{RESUMO}

Esta investigação apresenta uma metodologia para estimar a demanda de energia elétrica de edificações empregando modelos de cidades $3 D$. A idéia de incluir este tipo de dados está baseada no fato de estes dados serem ricos em informação geométrica e semântica, que pode ser útil no pronóstico de demanda de energia. Inicialmente apresentam-se os conceitos básicos de Sistemas de Informação Geográfica e energia elétrica; a seguir, escolhe-se o método que melhor se ajuste aos requerimentos do projeto.

Depois, apresentam-se as ferramentas de processamento para arquivos CityGML, continuando com a metodologia para estimar a demanda de energia elétrica concluindo com a presentação de um diagrama de atividades que expressa de melhor maneira as idéias propostas. Depois apresentam-se os resultados em uma área de estudo empregada durante a implementação. A secção final de este documento está dedicada às conclusões.

\section{PALAVRAS-ChAVE}

Demanda de Energia Elétrica, Modelos de Cidades 3D, Sistemas de Informação Geográfica, CityGML 


\section{Introducción}

E n la actualidad, nuevas tecnologías son desarrolladas diariamente en múltiples áreas de la ciencia, que van desde el Internet y teléfonos inteligentes, hasta carros eléctricos teniendo en común, que todos requieren energía eléctrica para su funcionamiento. Este requerimiento y el incremento de la preocupación por el medio ambiente, llevan a los planeadores a demandar un conocimiento detallado del consumo de energía eléctrica de un lugar (ciudad, región, país). Por este motivo, varias investigaciones se han desarrollado para la estimación del consumo de energía con diferentes propósitos como son, el pronóstico de energía o la influencia del comportamiento humano, solo para mencionar algunos.

Sin importar el modelo de estimación empleado, este proceso meticuloso y riguroso requiere de datos detallados, los cuales deben incluirse dentro de los resultados de la simulación del lugar, a fin de evaluar de una manera más real los requerimientos de energía (Fujimoto, Shimoda y Yamaguchi, 2011; Lee, Yi y Malkawi, 2011).

Se espera que un modelo 3D correctamente definido y detallado desde las perspectivas geométricas y semánticas conlleve a un mejor análisis de las aplicaciones energéticas de una unidad residencial. El conocimiento de los espacios de un edificio tiene una influencia directa en los requerimientos de equipamiento, llevando a una mejor definición de la base de datos de las aplicaciones energéticas (ejemplo: una cocina requiere estufas, hornos, etc.). Por tal motivo, se desarrolló esta investigación con la esperanza de que la estimación del consumo de energía eléctrica de un edificio residencial pueda ser hecho basado en su modelo 3D CityGML. 


\section{Sistemas de información geográfica}

I primer sistema de información geográfica SIG, apareció a finales de los años 1960 como una iniciativa del gobierno canadiense para producir mapas de capacidad del terreno, siendo analizados posteriormente para obtener información de rehabilitación de algunas granjas marginales (Coppock y Rhind, 1991). Desde sus orígenes, los SIG han sido empleados como sistemas computacionales para información geográfica que permiten procesar grandes cantidades de datos, los cuales están espacialmente localizados en un lugar específico.

En la actualidad, la mayoría de la información disponible está en 3D, esta clase de datos contiene no solo coordenadas $\mathrm{x}, \mathrm{y}$, sino también valores de altura (coordenada $z$ ) emergiendo nuevas posibilidades para que los usuarios representen el paisaje más allá de los modelos digitales de elevación (DEM en inglés) y modelos digitales de terreno (DTM en inglés) si además de construcciones humanas (puentes, túneles, edificios, etc.). Esta clase de datos, han sido modelados por muchos años empleando el Software CAD, como AutoCAD, Microstation o ArchiCAD. Sin embargo, se requieren nuevos estándares para que los datos puedan ser leídos, compartidos y empleados entre usuarios por múltiples plataformas. Uno de estos es CityGML, cuya funcionalidad es mucho más amplia que la simple representación semántica y geométrica de objetos 3D. CityGML (Lee, 2004) también puede ser empleado en proyectos como (Lee, 2004), para modelar la actividad humada usando SIG 3D o como fuente de datos para navegación en interiores (Nagel, et al, 2010).

\subsection{City Geography Markup Language}

El Lenguaje de Marcación Geográficao de Ciudades (CityGML), ha sido desarrollado desde 2002, siendo iniciativa del instituto de datos espaciales geográficos alemán (GDI-DE) y adoptado como estándar del Open 
Geospatial Consortium (OGC) en 2008, versión 1.0.0. En 2012 se actualizó el estándar a la versión 2.0.0, una revisión profunda que incluye "adiciones sustanciales y nuevas entidades al modelo temático de CityGML” (Kolbe, Gröger, Nagel y Häfele, 2012). El estándar fue implementado como un esquema de aplicación de GML versión 3.1.1 (GML3) el cual está basado en el modelo ISO 19107.

El estándar modela datos vectoriales 3D con su información semántica asociada (Kolbe, Gröger, Nagel y Häfele, 2012), también provee un mecanismo de extensión para enriquecer los datos con entidades identificables bajo la preservación de interoperabilidad semántica. CityGML presenta un modelo multiescalar con cinco niveles de detalle bien definidos (LOD), donde los objetos son más detallados con el incremento del LOD, tanto en geometría como en diferenciación geométrica. Un archivo CityGML, puede contener múltiples representaciones y geometrías para cada objeto en diferentes LOD simultáneamente (Tabla 1).

Tabla 1. LOD 0-4 de CityGML con sus requerimientos de exactitud propuestos

\begin{tabular}{|c|c|c|c|c|c|}
\hline & LODO & LOD1 & LOD2 & LOD3 & LOD4 \\
\hline $\begin{array}{l}\text { Descripción de } \\
\text { la escala }\end{array}$ & $\begin{array}{l}\text { Regional, } \\
\text { paisaje }\end{array}$ & $\begin{array}{l}\text { Región, } \\
\text { ciudad }\end{array}$ & $\begin{array}{l}\text { Ciudad, } \\
\text { distritos, } \\
\text { proyectos }\end{array}$ & $\begin{array}{l}\text { Distrito, mode- } \\
\text { los arquitectó- } \\
\text { nicos (exterior) } \\
\text { paisajes }\end{array}$ & $\begin{array}{l}\text { Modelos } \\
\text { arquitectó- } \\
\text { nicos } \\
\text { (interior) }\end{array}$ \\
\hline $\begin{array}{l}\text { Tipo de exacti- } \\
\text { tud }\end{array}$ & La más baja & Baja & Media & Alta & Muy alta \\
\hline $\begin{array}{l}\text { Exactitud abso- } \\
\text { luta de un punto } \\
\text { en 3D (posición } \\
\text { / altura) }\end{array}$ & $\begin{array}{l}\text { Más baja que } \\
\text { LOD1 }\end{array}$ & $5 / 5 \mathrm{~m}$ & $2 / 2 m$ & $0.5 / 0.5 \mathrm{~m}$ & $0.2 / 0.2 \mathrm{~m}$ \\
\hline Generalización & $\begin{array}{l}\text { Máxima } \\
\text { generali- } \\
\text { zación }\end{array}$ & $\begin{array}{l}\text { Bloques de } \\
\text { objetos como } \\
\text { entidades } \\
\text { generaliza- } \\
\text { das; } \\
>6^{*} 6 \mathrm{~m} / 3 \mathrm{~m}\end{array}$ & $\begin{array}{l}\text { Objetos } \\
\text { como entida- } \\
\text { des generali- } \\
\text { zadas; } \\
>4{ }^{*} 4 \mathrm{~m} / 2 \mathrm{~m}\end{array}$ & $\begin{array}{l}\text { Objetos como } \\
\text { entidades } \\
\text { reales; } \\
>2{ }^{*} 2 \mathrm{~m} / 1 \mathrm{~m}\end{array}$ & $\begin{array}{l}\text { Elementos } \\
\text { constructivos } \\
\text { y entradas } \\
\text { son repre- } \\
\text { sentados }\end{array}$ \\
\hline Instalaciones & no & No & yes & $\begin{array}{l}\text { Representación } \\
\text { de entidades } \\
\text { exteriores }\end{array}$ & $\begin{array}{l}\text { Objetos en } \\
\text { su forma real }\end{array}$ \\
\hline $\begin{array}{l}\text { Representación } \\
\text { del techo }\end{array}$ & si & Plano & $\begin{array}{l}\text { Estructuras } \\
\text { diferencia- } \\
\text { das }\end{array}$ & $\begin{array}{l}\text { Objetos con su } \\
\text { forma real }\end{array}$ & $\begin{array}{l}\text { Objetos con } \\
\text { su forma real }\end{array}$ \\
\hline $\begin{array}{l}\text { Piezas sobre- } \\
\text { salientes del } \\
\text { tejado }\end{array}$ & $\mathrm{Si}$ & No & $\begin{array}{l}\mathrm{Si}, \text { si se } \\
\text { conoce }\end{array}$ & yes & yes \\
\hline
\end{tabular}


Tabla 1. LOD 0-4 de CityGML con sus requerimientos de exactitud propuestos (continuación).

\begin{tabular}{|l|l|l|l|l|l|}
\hline $\begin{array}{l}\text { Mobiliario de la } \\
\text { ciudad }\end{array}$ & No & $\begin{array}{l}\text { Objetos } \\
\text { importantes }\end{array}$ & $\begin{array}{l}\text { Prototipos, } \\
\text { objetos } \\
\text { genera- } \\
\text { lizados }\end{array}$ & $\begin{array}{l}\text { Objetos con su } \\
\text { forma real }\end{array}$ & $\begin{array}{l}\text { Objetos con } \\
\text { su forma real }\end{array}$ \\
\hline $\begin{array}{l}\text { Objetos vegeta- } \\
\text { les solitarios }\end{array}$ & No & $\begin{array}{l}\text { Objetos } \\
\text { importantes }\end{array}$ & $\begin{array}{l}\text { Prototipos, } \\
\text { más de } 6 \mathrm{~m}\end{array}$ & $\begin{array}{l}\text { Prototipos, más } \\
\text { de } 2 \mathrm{~m}\end{array}$ & $\begin{array}{l}\text { Prototipos, } \\
\text { objetos con } \\
\text { su forma real }\end{array}$ \\
\hline $\begin{array}{l}\text { Cobertura de las } \\
\text { plantas }\end{array}$ & No & $>50 * 50 \mathrm{~m}$ & $>5 * 5 \mathrm{~m}$ & $<$ LOD2 & $<$ LOD2 \\
\hline
\end{tabular}

Fuente. Kolbe, Gröger, Nagel y Häfele, 2012.

Figura 1. Cinco niveles de detalle (LOD) definidos por CityGML

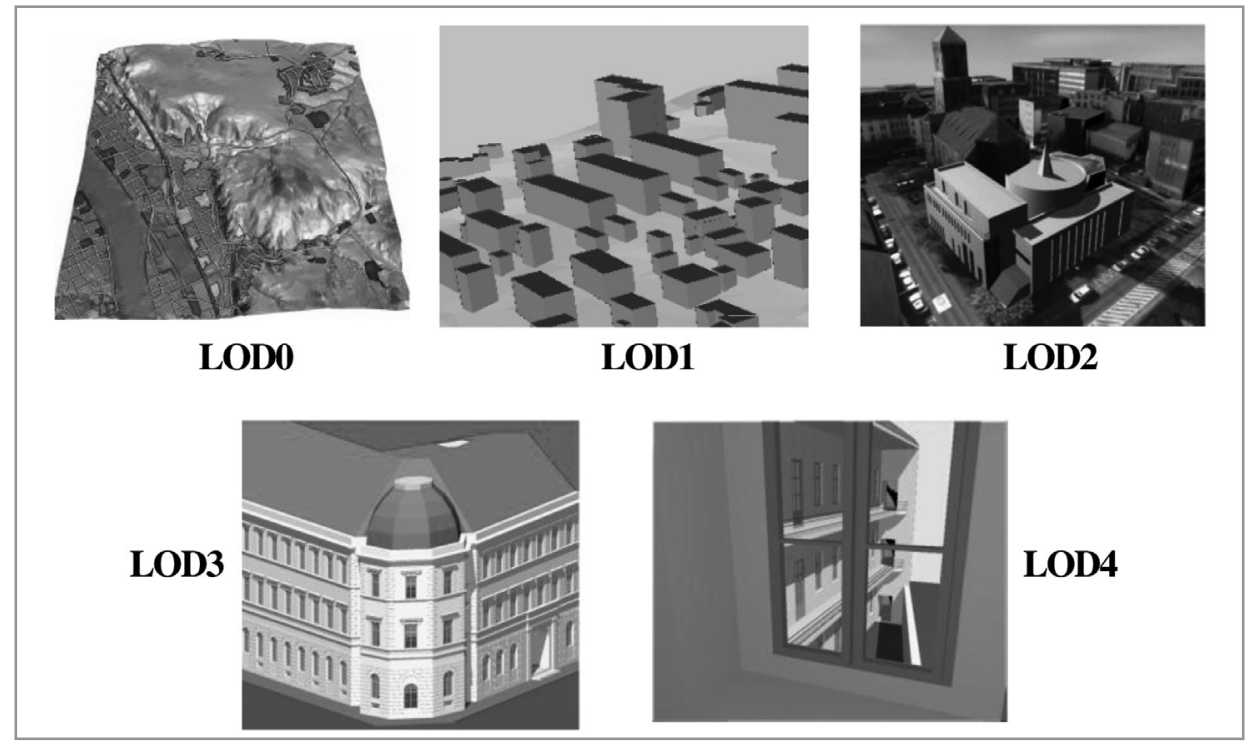

Fuente. OGC City Geography Markup Language (CityGML) Encoding Standard

\subsection{Modelo temático}

El estándar declara modelos temáticos permitiendo a CityGML un modelamiento explícito de cierto tipo de objetos, con el fin de alcanzar un alto nivel de definiciones e interoperabilidad entre aplicaciones. Incluye los siguientes módulos de extensiones temáticas: apariencias (Appearance), edificios (Building), mobiliario (CityFurniture), grupos de objetos (CityObjectGroups), genéricos (Generics), uso del suelo (LandUse), relieve 
(Relief), transporte (Transportation), vegetación (Vegetation), cuerpos de agua (WaterBodiesy), texturas de superficies (TexturedSurfaces), puentes (Bridges), túneles (Tunnels) (Kolbe, Gröger, Nagel y Häfele, 2012).

\subsubsection{Modelo temático edificios}

Este modelo es de especial relevancia debido al objetivo del proyecto, porque permite mostrar la representación de las edificiosaciones en todos los niveles de representación (LOD0 a LOD4); de igual forma permite la representación de un edificio a partir de un solo objeto o como la representación de las relaciones complejas entre las partes, por ejemplo, un edificio que consta de tres partes, una casa principal, un garaje y un depósito ( Figura 2).

Figura 2. Niveles de detalle del modelo temático edificios de CityGML

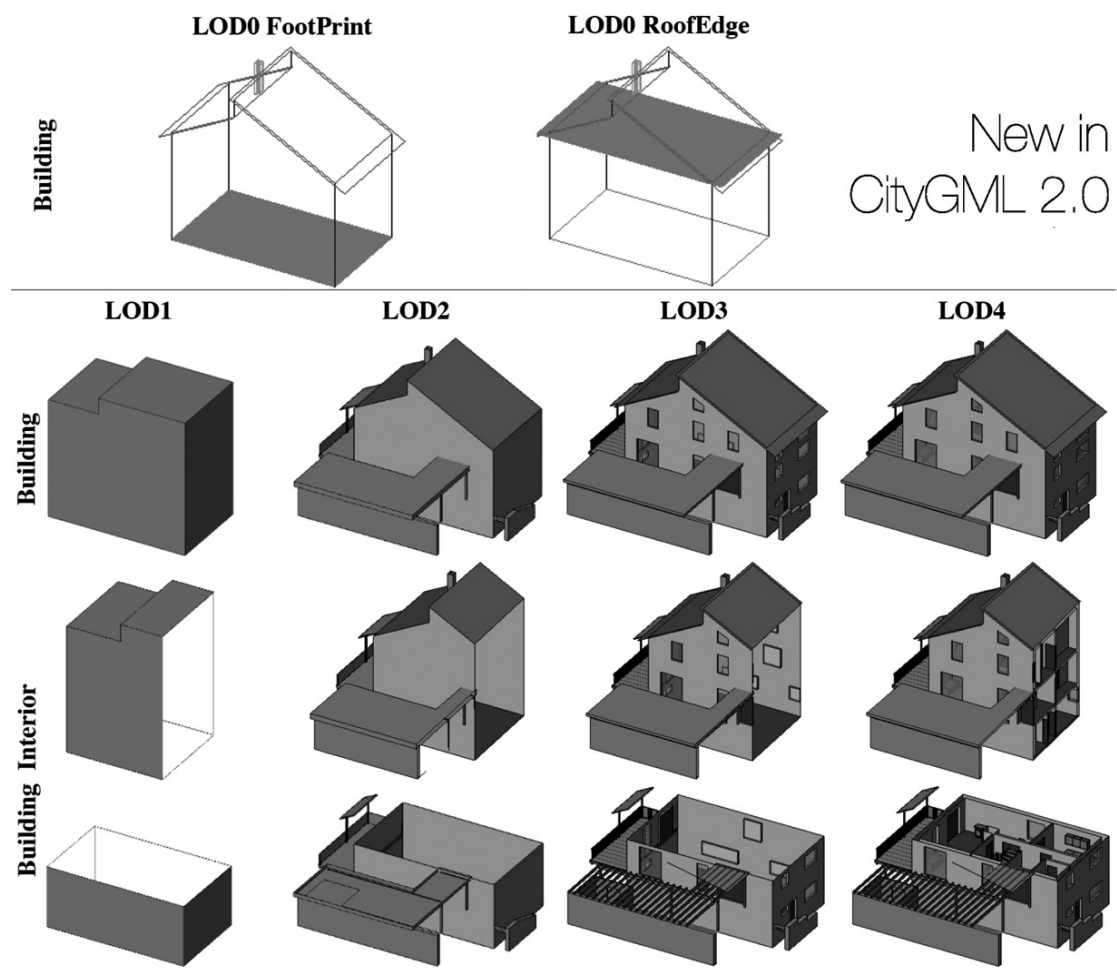

Fuente. Eicker, Nouvel, Schulte, Schumacher y Coors (2012). 


\section{Energía}

T a energía eléctrica, es relevante para el objetivo de este proyecto y es definida como "la energía hecha disponible por el flujo del cambio eléctrico a través de un conductor" (Princenton University, 2012); así, entre más alto sea el voltaje, más energía eléctrica estará disponible.

La generación y utilización de energía incluye la conversión de una a otra, que en muchas situaciones requiere de pasos intermedios. La energía puede ser clasificada en primaria, secundaria final y útil. ( Tabla 2)

Tabla 2. Flujo de energía

\begin{tabular}{|l|l|l|}
\hline \multicolumn{1}{|c|}{ Energía } & \multicolumn{1}{|c|}{ Tecnología } & \multicolumn{1}{c|}{ Ejemplos } \\
\hline Primaria & & $\begin{array}{l}\text { Carbón, madera, agua, estiércol, petróleo, } \\
\text { etc. }\end{array}$ \\
\hline & Conversión & Planta de poder, horno, refinería, digestor. \\
\hline Secundaria & & Refinería petrolera, electricidad, biogás. \\
\hline & $\begin{array}{l}\text { Transporte / } \\
\text { transmisión }\end{array}$ & Camiones, oleoductos, cables. \\
\hline Final & & $\begin{array}{l}\text { Diesel, carbón de leña, electricidad, } \\
\text { biogás. }\end{array}$ \\
\hline & Conversión & Mmotores, calentadores, estufas. \\
\hline Útil & & Ejes, calefacción. \\
\hline
\end{tabular}

Fuente. Energy for Sustainable Rural Development Projects ( 1991).

- Energía primaria: es la energía como se encuentra disponible en el ambiente natural, ej. primera fuente de energía .

- Energía secundaria: es la energía lista para ser transportada o transmitida.

- Energía final: es la energía que es consumida o comprada por los receptores. 
- Energía útil: es la energía empleada como fuente para otra aplicación de usuario-final.

La energía eléctrica, la cual es de relevancia para el objetivo de este proyecto, es definida como " la energía hecha disponible por el flujo del cambio eléctrico a través de un conductor" (Princenton University, 2012), así entre más alto sea el voltaje, más energía eléctrica estará disponible.

\subsection{Metodologías empleadas para la estima- ción de la demanda de energía eléctrica}

Se presentan los pros y contras de diferentes métodos empleados para la predicción del consumo de energía, teniendo en cuenta que el propósito de este proyecto, es emplear modelos 3D de ciudades como fuente principal para la estimación del consumo de energía de un edificio (Tabla 3).

Tabla 3. Ventajas y desventajas de los métodos para pronosticar la demanda de energía

\begin{tabular}{|c|c|c|}
\hline Método & Ventajas & Desventajas \\
\hline Tendencia & $\begin{array}{l}\text { - Pocos conocimientos } \\
\text { requeridos. } \\
\text { - Rápido y no costoso. } \\
\text { - Actualizable a través de ajuste } \\
\text { de los datos. } \\
\text { - Requerimientos de datos } \\
\text { mínimos. }\end{array}$ & $\begin{array}{l}\text { - Vulnerable a cambios. } \\
\text { - No es explicito para auditar errores. }\end{array}$ \\
\hline $\begin{array}{l}\text { Series de } \\
\text { tiempo }\end{array}$ & $\begin{array}{l}\text { - Exacto para periodos de tiempo } \\
\text { cortos. } \\
\text { - Bajo costo. } \\
\text { - Requerimientos de datos míni- } \\
\text { mos. } \\
\text { - Evaluación estadística de la } \\
\text { incertidumbre del pronóstico. }\end{array}$ & $\begin{array}{l}\text { - Requiere un conjunto de datos his- } \\
\text { tórico grande. } \\
\text { - No trata los factores explícitamente. } \\
\text { - Interpretación difícil de los errores. }\end{array}$ \\
\hline
\end{tabular}


Tabla 3. Ventajas y desventajas de los métodos para pronosticar la demanda de energía (Continuación).

\begin{tabular}{|c|c|c|}
\hline Usuario -final & $\begin{array}{l}\text { - Puede rastrear la verdadera } \\
\text { localización de los errores de la } \\
\text { predicción. } \\
\text { - Requiere conocimientos inter- } \\
\text { medios técnicos y computacio- } \\
\text { nales. } \\
\text { - Resultados fáciles de explicar. }\end{array}$ & $\begin{array}{l}\text { - Requiere una gran cantidad de } \\
\text { datos detallados. } \\
\text { - El ensamblaje de datos es costoso } \\
\text { y difícil. } \\
\text { - Tecnología debe ser especificada } \\
\text { explícitamente. } \\
\text { - Requiere conocimientos de las } \\
\text { tecnologías y usos del usuario final. }\end{array}$ \\
\hline Econométrico & $\begin{array}{l}\text { - Provee evaluaciones estadís- } \\
\text { ticas de la incertidumbre del } \\
\text { pronóstico. } \\
\text { - Combina información econó- } \\
\text { mica y demográfica en el } \\
\text { territorio en servicio. } \\
\text { - Puede incorporar otros métodos. } \\
\text { - Modelos pueden ser fácilmente } \\
\text { re-estimados. }\end{array}$ & $\begin{array}{l}\text { - Requiere conocimientos y expe- } \\
\text { riencia en econometría. } \\
\text { - Requiere gran cantidad de datos } \\
\text { detallados para desagregar el } \\
\text { modelo. } \\
\text { - Costos pueden ser relativamente } \\
\text { altos. }\end{array}$ \\
\hline
\end{tabular}

Fuente. Mehra y Bharadwaj (2000), Mitchell, et al ( 1986); Cullen (1999).

Con base a la motivación de esta investigación (sección 3.1) y la tabla 3 , se seleccionó el método Usuario-final como método de predicción. Sin embargo, es importante hacer hincapié en que este método requiere una base de datos detallada que incluye tantos datos de las aplicaciones energéticas como sea posible. Este tipo de datos está fuertemente relacionado con lo que se llama en esta investigación influencia de los ocupantes o como algunos autores consideran, comportamiento humano, el cual será tratado en la sección 3.2., (Page, Robinson, \& Scartezzini, 2007; Shuvra, Rahman, Ali, \& Khan, 2011; Santin, Itard, \& Visscher, 2009), consideran comportamiento humano.

\subsection{Influencia de los ocupantes}

El comportamiento humano tiene un gran impacto en el uso de energía, siendo relevante junto con otros parámetros, tales como equipos y aplicaciones (Santin, Itard y Visscher, 2009), causando variaciones dramáticas en el consumo de energía en unidades de vivienda familiar con las mismas características físicas (Branco, Lachal, Gallinelli y Weber, 2004). Normalmente, se incluye en los modelos como patrones de ocupación que representan un usuario promedio (Fujimoto, Shimoda y Yamaguchi, 2011) 
o por horarios de operación (Page, Robinson y Scartezzini, 2007; Lee, Yi y Malkawi, 2011).

Otra característica que se distingue en varios estudios (Reinhart, 2004; Wout, Dirk y Hugo, 2010; Roetzel, Dietrich, Tsangrassoulis, \& Busching, 2010) es que el comportamiento de los usuarios se puede considerar como pasivos o activos, con base en la dinámica en que se encienden/apagan los dispositivos según influencias externas por ejemplo la luz del día, siendo los pasivos aquellos que solo encienden el dispositivo cuando llegan y lo apagan al salir. Debido a la dificultad de modelar comportamientos estocásticos y muy dinámicos, algunos autores como (Clevenger \& Haymaker, 2006) han incluido en sus estudios el comportamiento humano en condiciones extremas, llamándolas mejor y peor escenario dependiendo del tiempo de uso.

\subsection{Aplicaciones de energéticas}

Después de presentar el modelo de predicción elegido (sección 3.4.1), es necesario especificar las diferentes aplicaciones energéticas que son consideradas en esta investigación. Sin embargo, este método requiere una gran base de datos a fin de producir mejores resultados. Finalmente, el consumo de energía eléctrica en una unidad residencial puede ser expresado como la sumatoria de las aplicaciones energéticas ( Ecuación 1).

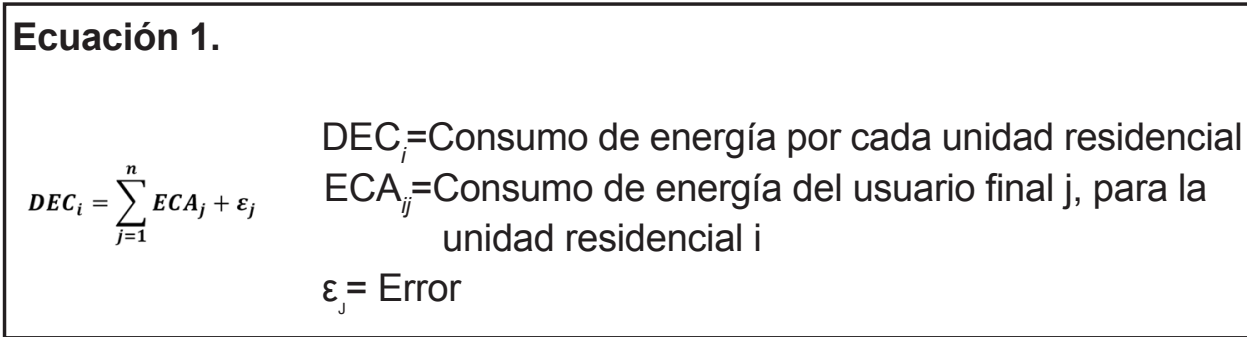

Hay que considerar que el incremento en el número de usuarios en una vivienda, no se traduce en un incremento lineal en el consumo de energía (Haldi y Robinson, 2010; Fujimoto, Shimoda, y Yamaguchi, 2011). Por tal razón, los usuarios son incluidos en la estimación de la energía como el logaritmo de su número, garantizando así un incremento en el consumo de energía de esa aplicación (Kema-Xenergy, 2004), (Kema, 2010) (KEMAXENERGY, 2004; KEMA, Inc., 2010) (Ecuación 2): 


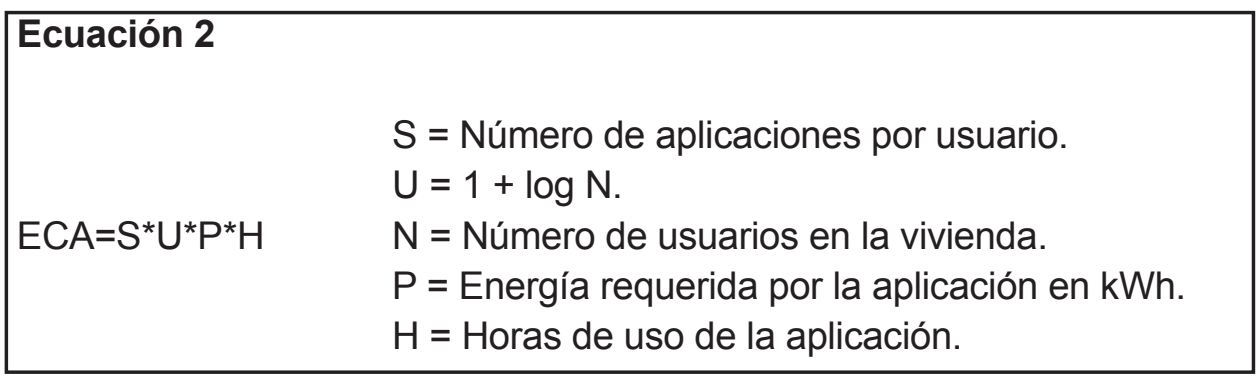

El grupo de aplicaciones energéticas del método Usuario-final consideradas para esta investigación y que fueron definidas basadas en (Meier, Rainer y Greenberg, 1992; Kema-Xenergy, 2004; KEMA, Inc., 2010) (Meier, Rainer, y Greenberg, 1992; KEMA-XENERGY, 2004; KEMA, Inc., 2010) (Meier, Rainer, y Greenberg, 1992; Kema-Xenergy, 2004; KEMA, Inc., 2010) son presentadas a continuación.

- Preparación de comida.

- Lavado.

- Energía eléctrica.

- Entretenimiento y tecnología.

- Computador personal y oficinas en casa.

- Misceláneos. 


\section{Herramientas completas para procesar archivos \\ CityGML}

L os conceptos presentados en las secciones previas, son la base para el desarrollo de esta investigación, sin embargo, se requiere una implementación física la cual se lleva a cabo empleando un modelo 3D de ciudad basado en el estándar CityGML. Esto requiere un marco de implementación que incluye el uso de diferentes herramientas tecnológicas con las cuales se ejecutará el diagrama de actividades.

- Citygml4j 2.0ea. API y librería de clases de Java desarrollado por el Instituto Tecnológico de Berlin (TU Berlin), que permite manipular archivos de datos CityGML. (IGGS, 2009).

- JTS Topology Suite 1.13. API para el procesamiento de geometrías usando Java.

- FME. Es una colección de herramientas espaciales para extraer, transformar y cargar múltiples formatos, la cual es útil para la manipulación y transformación de datos espaciales (Carriòn, 2010).

- ArcGIS. Software para información geográfica de ESRI. Plataforma empleada para la visualización de datos y presentación de resultados. 


\section{Metodología}

Tr a estimación del consumo de energía fue dividida en tres partes principales; la primera, es la estimación de los parámetros del edificio (incluyendo número de habitantes); la segunda, se refiere a las aplicaciones energéticas la cual indica que equipamiento es considerado; la última, está enfocada en el comportamiento humano, en este punto se presenta el modelo de vida empleado en el proyecto.

\subsection{Requerimientos de datos del edificio}

Es fundamental tener un modelo 3D detallado de un edificio para cumplir el objetivo de esta investigación, ya que puede proveer información relevante como el número de pisos, cantidad de unidades residenciales y de alcobas. El estándar CityGML, provee el escenario ideal para este tipo de modelamiento. Se presenta la relación de los temas semánticos que están disponibles para el modelo de edificios y el nivel de detalle que se encuentran disponibles.

Tabla 4. Temas semánticos de la clase AbstractBuilding

\begin{tabular}{|l|l|l|l|l|l|}
\hline \multicolumn{1}{|c|}{ Tema geométrico/semántico } & \multicolumn{1}{|l|}{ LOD0 } & LOD1 & LOD2 & LOD3 & LOD4 \\
\hline Base y techo del edificio & $\mathrm{X}$ & & & & \\
\hline Forma volumétrica del edificio & & $\mathrm{X}$ & $\mathrm{X}$ & $\mathrm{X}$ & $\mathrm{X}$ \\
\hline Superficies envolventes del edificio & & $\mathrm{X}$ & $\mathrm{X}$ & $\mathrm{X}$ & $\mathrm{X}$ \\
\hline Curvas de intersección del edificio & & $\mathrm{X}$ & $\mathrm{X}$ & $\mathrm{X}$ & $\mathrm{X}$ \\
\hline Partes curvas envolventes del edificio & & & $\mathrm{X}$ & $\mathrm{X}$ & $\mathrm{X}$ \\
\hline Partes de la construcción & & $\mathrm{X}$ & $\mathrm{X}$ & $\mathrm{X}$ & $\mathrm{X}$ \\
\hline Superficies limítrofes & & & $\mathrm{X}$ & $\mathrm{X}$ & $\mathrm{X}$ \\
\hline Otras instalaciones del edificio & & & $\mathrm{X}$ & $\mathrm{X}$ & $\mathrm{X}$ \\
\hline Aperturas & & & & $\mathrm{X}$ & $\mathrm{X}$ \\
\hline Salones & & & & & $\mathrm{X}$ \\
\hline Instalaciones en el interior del edificio & & & & & $\mathrm{X}$ \\
\hline
\end{tabular}

Fuente. Kolbe, Gröger, Nagel y Häfele (2012). 
Así como fue mencionado el objetivo de esta inves-tigación es estimar la demanda de energía eléctrica para edificios residenciales, esta información puede extraerse del atributo Function del archivo CityGML, el cual contiene el propósito del objeto modelado (IGGS, 2009). Debido a que el enfoque de la investigación es el modelo temático, edificios, este atributo indica si es residencial, público, industrial o comercial. El conjunto de datos empleado en esta investigación, corresponde al barrio de Charlottenburg (Berlín), incluyendo 833 objetos clasificados como edificios, el atributo Function incluye un código acorde al sistema de información catastral alemán ALK (Senatsverwaltung für Stadtentwicklung, 2005).

\subsubsection{Estimación del número de pisos por edificio}

Basados en el hecho que la representación LOD2 modela tan solo las características que definen un edificio,( Figura 3), se puede declarar que este está delimitado en el nadir y cenit por los objetos GroundSurface y RoofSurface respectivamente, así como que el GroundSurface es la huella del edificio o mejor, su área construida. Ya que un edificio puede estar constituido por varias partes, se concluye que la sumatoria de todas sus GroundSurfaces es el área bruta por piso del edificio.

Figura 3. Edificio en LOD2, superficies que lo delimitan (a), delimitación del espacio amigable, (b)

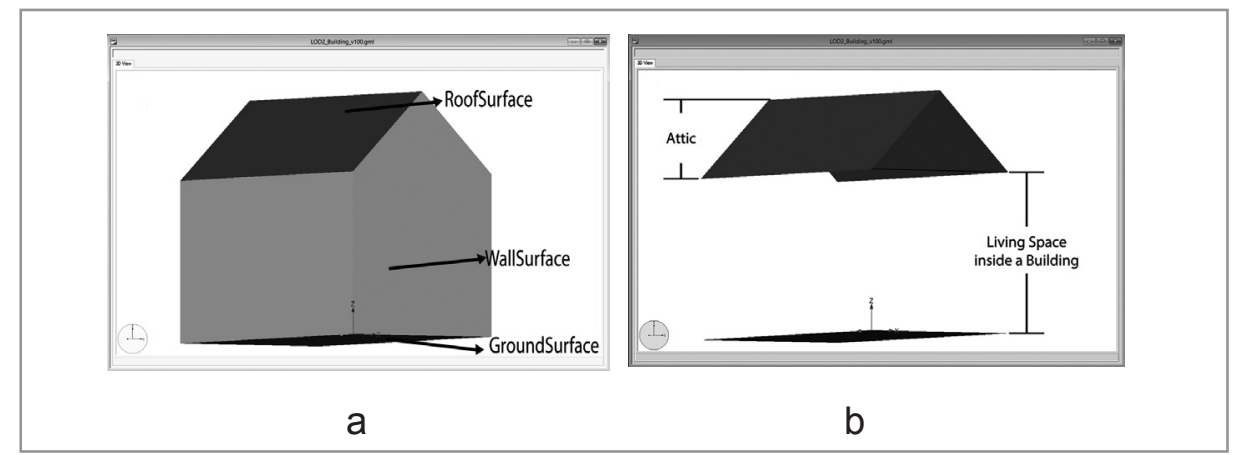

Fuente. Elaboración propia del autor, a partir de FZKViewer-2.3. 
A partir de la información anterior (Figura 3), se pueden extraer los siguientes datos:

\section{Ecuación 3. Altura del área habitable}

$$
B L S_{h}=R S z_{\text {min }}-G S z
$$

$B L S_{h}$ - Altura del espacio habitable.

$\mathrm{RSz}_{\text {min }}$ - Altura mínima del techo.

GSz - Altura de la base del edificio.

\section{Ecuación 4. Número de pisos por edificio}

$$
\text { NoS=int } \frac{\mathrm{BLS}_{\mathrm{h}}}{\mathrm{SS}_{\mathrm{h}}}
$$

NoS - Número de edificios.

$\mathrm{BLS}_{\mathrm{h}}$ - Altura del área habitable.

$\mathrm{SS}_{\mathrm{h}} \quad$ - Altura estándar de un piso.

De acuerdo con Neufert y et al (2005) la altura estándar de un piso es $2,75 \mathrm{~m}$, sin embargo, este valor y las aseveraciones hechas en este libro, están basadas en la normatividad vigente a la fecha de publicación, por tal motivo es válido por un periodo de tiempo específico, es asi como la altura de un piso puede variar según el año de construcción del edificio.

Por tal motivo, se realizó un muestreo dentro del área de estudio tomando el número de pisos por edificio y luego obteniendo las alturas promedio para cada caso ${ }^{1}$. A pesar que el modelo de datos incluye el campo año de construcción, este no estaba diligenciado, por tal motivo se requirió obtener esta información a través del WMS "Año de construcción del edificio 19921993" disponible para Berlín del servicio FIS-Broker (Senatsverwaltung für Stadtentwicklung und Umwelt, 2013), ( Figura 4). De igual forma, se tuvo en cuenta el espacio entre pisos el cual no ha sido concluido en la altura dce estyos, lo que significa $40 \mathrm{~cm}$ adicionales al valor promedio por piso según el año de construcción.

\footnotetext{
1 El muestreo incluye los datos de 144 edificios, incluyendo el conteo del número de pisos y el número total de timbres disponibles en las entradas, asumiendo que cada uno pertenece a una unidad residencial diferente. No hubo un criterio específico para el muestro, más caminar a través del área de estudio.
} 
Figura 4. WMS del año de construcción de los edificios en Berlín para el área de estudio
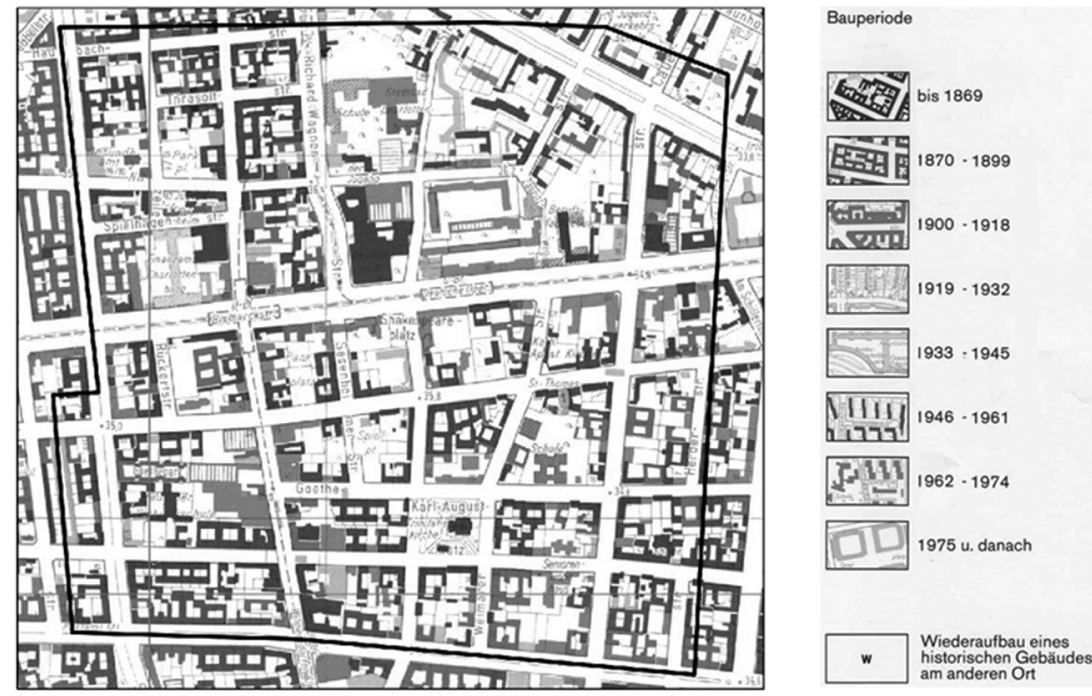

Fuente. Elaboración propia del autor, a partir de FIS-Broker WMS.

\subsubsection{Techos}

Es el último parámetro que debe ser considerado en esta sección, sin embargo, no hay alguna indicación que revele si un techo es habitable o no. En esta investigación, se tuvo en cuenta la normatividad de varios estados federales alemanes, indicando que un área es habitable si tiene un altura de al menos 2,30m (Bayerische Staatregierung, 2007; Hamburg Senat, 2005; Recht NRW, 2000) Por tal motivo, se incluirá un piso adicional al edificio si cumple lo presentado en la Ecuación 5.

\section{Ecuación 5. Altura del techo}

$$
\mathrm{Roof}_{\mathrm{h}}=\mathrm{RSz}_{\text {max }}-\mathrm{WSz} \text { max }
$$

Roof $_{h} \quad$ - Altura del techo.

$\mathrm{RSz}_{\text {max }}$ - Máximo valor de la coordenada $Z$ del techo.

$\mathrm{WSZ}_{\max }$ - Máximo valor de la coordenada $Z$ de las paredes. 


\subsubsection{2 Áreas públicas dentro del edificio}

Después de definir el número de pisos, es necesario extraer las áreas comunes del edificio; esta consideración es hecha por dos motivos, la primera es que, las áreas tienen diferentes aplicaciones energéticas y la segunda es que una vez que han eliminado, se puede asumir el restante del piso como áreas privadas o asignadas a vivienda. Esta clase de suposiciones no son necesarias en el momento de contar con un modelo con representación LOD4, ya que modela la parte interna del edificio hasta sus salones. Basado en (Loga, Diefenbach, Knissel y Born, 2005) se asume como el $25 \%$ del piso el área común del edificio.

\subsubsection{Número de unidades residenciales / habitantes}

Esta es la parte crítica de la investigación, varios autores (Wohnungsanwalt, 2009; Amt für Statistik Berlin-Brandenburg, 2011; DESTATIS, 2009), consideran que el área promedio de un apartamento para Berlín es $71 \mathrm{~m}^{2}$, más aún indican que el área promedio de una alcoba es $19.57 \mathrm{~m}^{2}$. Basado en Senatsverwaltung für Stadtentwicklung (2010) los datos estadísticos en Berlín son:

$70.4 m^{2}$ - área promedio por unidad residencial.

$38.8 \mathrm{~m}^{2}$ - Área promedio por persona.

1.82 - número de personas por unidad residencial.

Sin embargo, estos valores no representan el área de estudio ya que como se indica son para toda la ciudad. Finalmente, se hizo un promedio para cada categoría según el año de construcción del edificio.

\subsection{Flujo de trabajo para la estimación de los parámetros del edificio}

Se presenta el diagrama de actividades UML para la estimación de las propiedades del edificio en este estudio. 


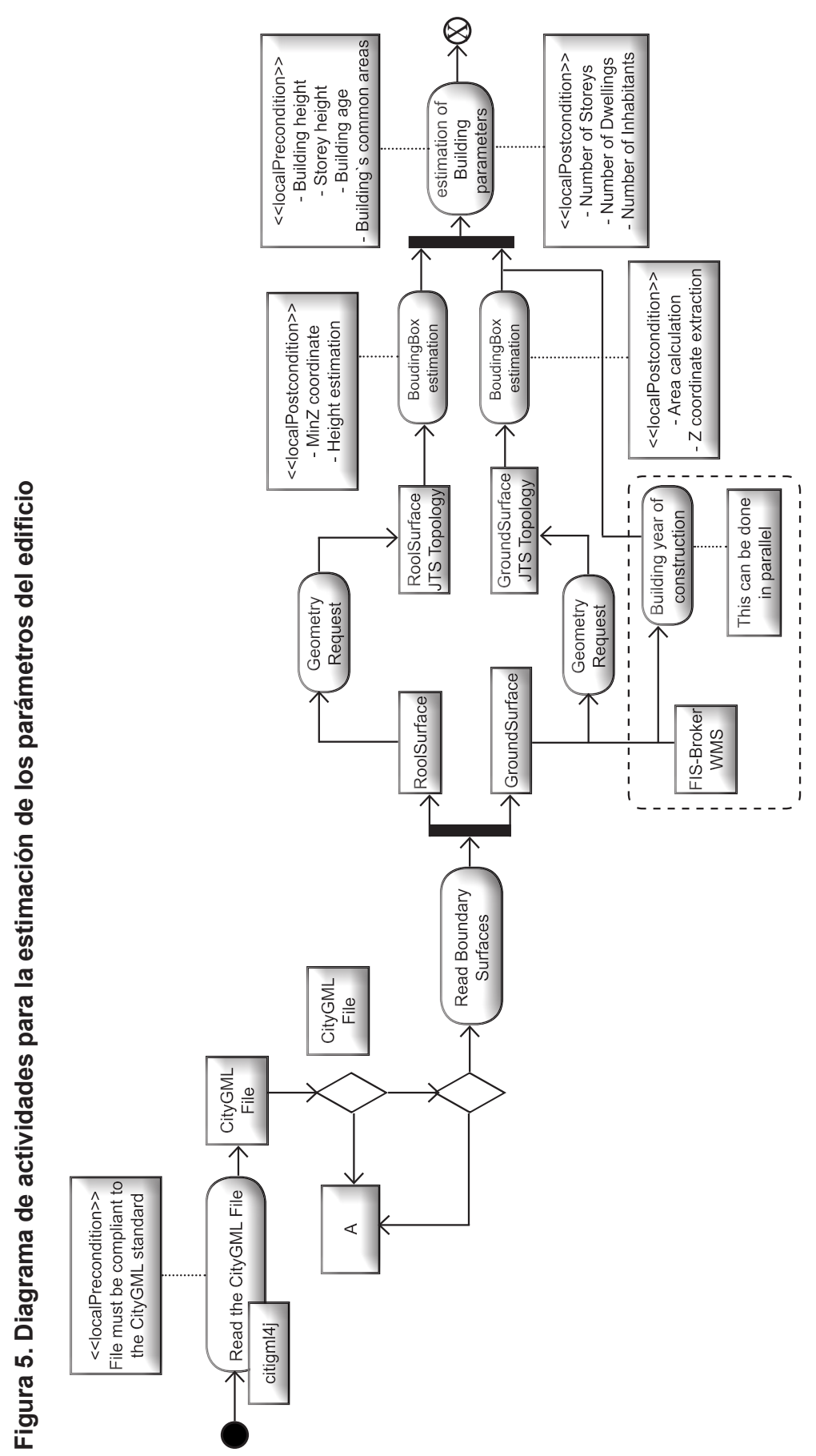

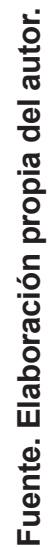




\subsection{Aplicaciones energéticas}

Esta sección, presenta los valores de consumo de energía del equipamiento considerado en este estudio para las aplicaciones energéticas contempladas con base al modelo Usuario-final seleccionado para el pronóstico del consumo de energía. (Tabla 5).

Tabla 5. Aplicaciones energéticas y su consumo de energía

\begin{tabular}{|l|l|}
\hline Aplicación & Consumo (kWh) \\
\hline Nevera & 0.35 / año \\
\hline Congelador & 0.25 / año \\
\hline Congelador / nevera & $0.408(1) /$ año \\
\hline Estufa (cuatro puestos) & 4.5 \\
\hline Horno & $1.56(1)$ \\
\hline Chimenea & 0.3 \\
\hline Horno microondas & 1.2 \\
\hline Lavaplatos eléctrico & $1.44(1)$ \\
\hline Lavadora & $0.63(1) /$ carga \\
\hline Secadora & $2.5 /$ carga \\
\hline \multicolumn{2}{|c|}{ Luz eléctrica (bombillas) } \\
\hline Bombilla 100 & 0.1 \\
\hline Bombilla 40 & 0.04 \\
\hline Bombilla 32 & 0.032 \\
\hline
\end{tabular}

\begin{tabular}{|l|l|}
\hline Aplicación & Consumo (kWh) \\
\hline Bombilla ahorradora & 0.02 \\
\hline TV & $\begin{array}{l}0.176(6) \\
0.001 \text { Standby }\end{array}$ \\
\hline Home Theater & $0.105(5)$ \\
\hline Video consolas & $\begin{array}{l}0.002 \text { Standby } \\
0.102 \text { on }\end{array}$ \\
\hline Computador & 0.2 \\
\hline Multifuncional & 0.01 \\
\hline Modem ADSL & 0.03 \\
\hline Celular & $0.005(4)$ \\
\hline Tablet & $0.012(4)$ \\
\hline Docking station & $0.007(3)$ \\
\hline Aspiradora & 1.3 \\
\hline Plancha & 1 \\
\hline Cafetera & 0.6 \\
\hline Adaptador TV digital & 0.01 \\
\hline
\end{tabular}

Fuente. Min.EnergiaMinas, (s.f.): Carbon Footprint Ltd., (s.f.); Stratitum Energy, (2010); Sony, ( 2013), Ho, (2009), Phillips, (2013); Bluejay, (2010).

En este estudio se consideró solo un dispositivo por aplicación exceptuando la luz eléctrica. El número de usuarios, es el resultado del proceso hecho.

\subsection{Comportamiento humano}

La propuesta de estilo de vida realizada en este estudio, dos clases de (día habil y fines de semana), siendo cada uno dividido en tres actividades principales: dormir, en casa y fuera de casa. El propósito de dividir el día fue el de presentar un marco de tiempo que indicará cuando los habitantes, 
estaban en su vivienda y en ese periodo de tiempo, debia existir una clara diferencia en el consumo de energía, partiendo del hecho en que la población está despierta o dormida.

Posterior a la declaración del marco de tiempo, se define un horario para cada una de las aplicaciones (Clevenger y Haymaker, 2006), a fin de analizar la influencia de la ocupación humana, siendo clasificado en tres rangos bajo, medio y alto (tabla 6 ) con base al tiempo de uso.

Figura 6. Propuesta de clasificación de la ocupación de los habitantes

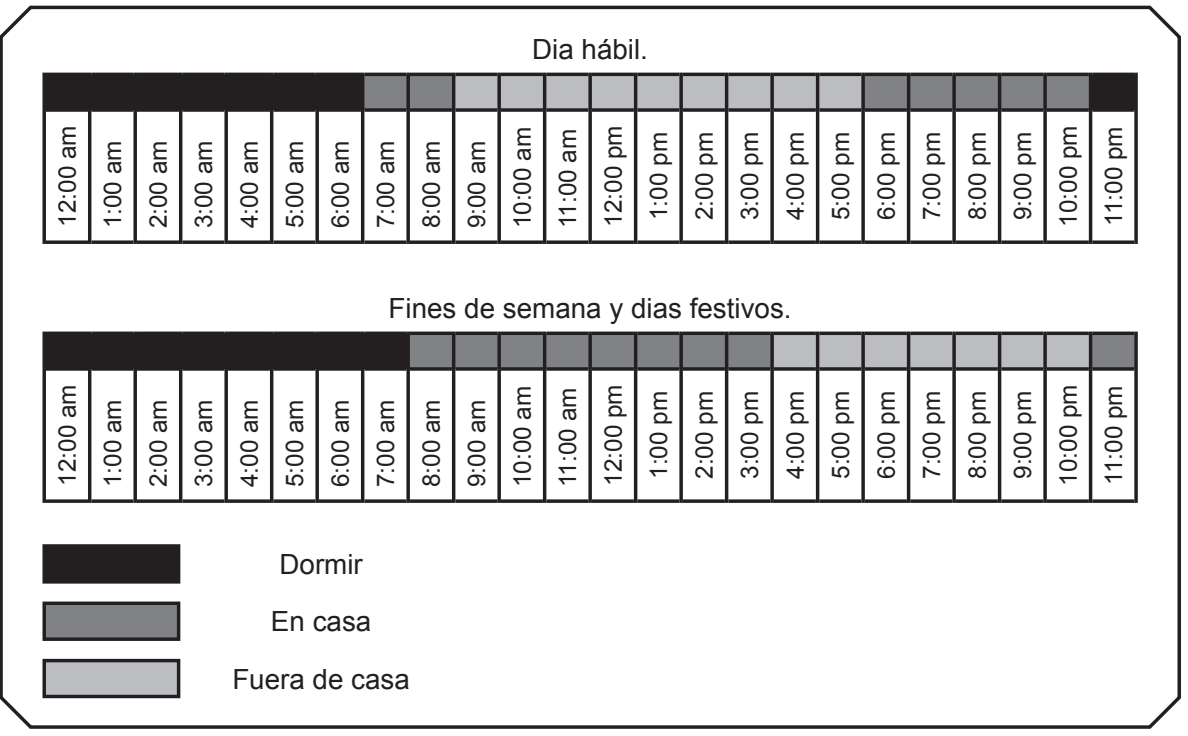

Fuente. Elaboración propia del autor.

Tabla 6. Rangos de horario por aplicación energética

\begin{tabular}{|l|c|c|c|}
\hline \multicolumn{1}{|c|}{ Aplicación } & Alto & Medio & Bajo \\
\hline Preparación de comida & $2 h$ & $1 h$ & $0.5 h$ \\
\hline Nevera/congelador & \multicolumn{3}{|c|}{ Todo el día } \\
\hline Lavandería & 2 cargas/semana & 1 carga/semana & 0.5 carga/semana \\
\hline Luz eléctrica & $16 h$ & $8 h$ & $4 h$ \\
\hline \multicolumn{4}{|c|}{ Entretenimiento y tecnología, computadores personales y misceláneos } \\
\hline Entresemana & $6 h$ & $3 h$ & $1.5 h$ \\
\hline Fin de semana & $8 h$ & $4 h$ & $2 h$ \\
\hline Hornos microondas & $20 \mathrm{~min}$ & $10 \mathrm{~min}$ & $5 \mathrm{~min}$ \\
\hline
\end{tabular}

Fuente. Elaboración propia del autor. 


\subsection{Flujo de trabajo para la estimación del consumo de energía}

Se presenta un diagrama UML para la estimación del consumo de energía de un edificio (Figura 7 ). Es una continuación de la figura 5, debido a que los parámetros del edificio son requeridos para este propósito.

Figura 7. Diagrama de actividades para la estimación del consumo de energía

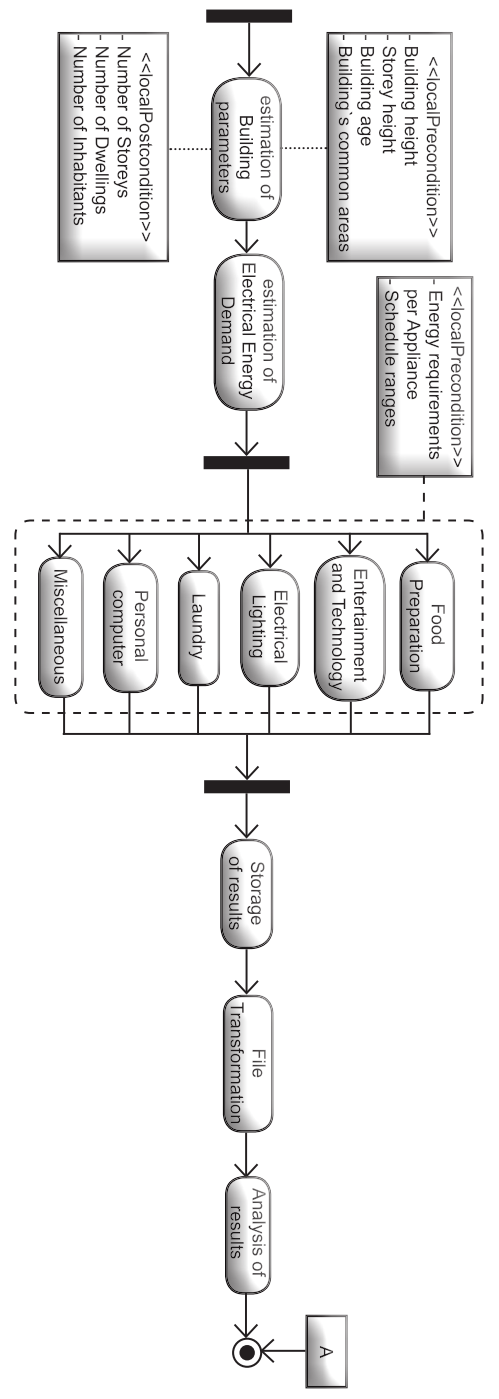

Fuente. Elaboración propia del autor. 


\section{Resultados}

on el propósito de tener mejor visualización de los resultados, se presentan los valores de tres edificios. Se emplearon dos parámetros para determinar los resultados obtenidos en las secciones, primero considerando un valor estándar para todos los edificios y el segundo, con base a la clasificación a partir de su año de construcción.

\subsection{Parámetros del edificio}

\subsubsection{Número de pisos por edificio}

Tabla 7. Comparación número de pisos por edificio según los dos métodos empleados

\begin{tabular}{|l|c|c|c|c|c|c|}
\hline \multicolumn{1}{|c|}{ ID Edificio } & Pisos & SStd.H & Dif & $\begin{array}{c}\text { Año } \\
\text { construcción }\end{array}$ & SH,YoC & Dif \\
\hline $\begin{array}{l}\text { BLDG } \\
0003000000067203\end{array}$ & 7 & 9 & -2 & 1961 & 9 & -2 \\
\hline $\begin{array}{l}\text { BLDG } \\
\text { 0003000000069010 }\end{array}$ & 6 & 9 & -3 & 1899 & 7 & -1 \\
\hline $\begin{array}{l}\text { BLDG } \\
\text { 000300000006f0e2 }\end{array}$ & 6 & 8 & -2 & 1899 & 6 & 0 \\
\hline
\end{tabular}

Fuente. Elaboración propia del autor.

Siendo:

Pisos - Valor recogido en el muestreo hecho en el área de estudio.

sStd.H - Altura estándar del piso.

${ }^{\mathrm{s}} \mathrm{H}$, YoC - Altura estándar por piso según el año de construcción.

Es posible identificar el gran impacto que tiene la discriminación de los edificios a partir del año de construcción, reduciendo el error con respecto a la muestra tomada (Tabla 7). Finalmente, se realizó el test estadístico $x^{2}$ (Greenwood y Nikulin, 1996), a fin de evaluar la calidad del ajuste de los datos con base en la ecuación 6 . 


\section{Ecuación 6. Test estadístico $\mathrm{x}^{2}$}

$$
x^{2}=\sum_{i=1}^{n} \frac{\left(O_{i}-E_{i}\right)^{2}}{E_{i}}
$$

$\mathrm{X}^{2}$ - test estadístico acumulativo el cual se aproxima asintóticamente a una distribución normal.

$\mathrm{O}_{\mathrm{i}}$ - valor observado.

$\mathrm{E}_{\mathrm{i}}$ - valor esperado.

$\mathrm{N}$ - número de observaciones.

Teniendo un nivel de confianza del $95 \%$ y un grado de libertad de 143 , el valor criticocrítico $\mathrm{T}_{x}{ }^{2}=171.907$ se aceptará la hipótesis nula Ho si se cumple $q \alpha, f<T_{x}{ }^{2}$ ). Los resultados obtenidos fueron:

$$
\begin{array}{ll}
\mathrm{S}_{\mathrm{Std.H}}: 62.8764<171.907 & \begin{array}{l}
\text { Para los dos métodos se acepta la } \\
\mathrm{S}_{\mathrm{H}, \mathrm{YoC}}: 19.955<171.907
\end{array} \\
& \begin{array}{l}
\text { hipótesis nula, indicando que ambos } \\
\text { siguen una distribución normal. }
\end{array}
\end{array}
$$

A pesar que el error fue minimizado, este se sigue presentando en muchos edificios, debido al altillo ya que no existe un parámetro físico que determine si un techo puede ser usado como residencial o no. Muchas construcciones presentan un techo que es reconocido por la metodología empleada en este estudio como habitable, mas no es usado por alguna razón conocida.

\subsubsection{Número de unidades residenciales por edificio}

Inicialmente, se estimó el área promedio de un apartamento en el área de estudio empleando la ecuación 7 . se obtuvo un valor promedio de $82.34 \mathrm{~m}^{2}$ lo cual difiere completamente respecto al valor promedio de Berlín que es $70.4 \mathrm{~m}^{2}$. Por tal razón, se decidió realizar la estimación del número de apartamentos discriminando un área promedio por cada uno, según el año de construcción del edificio. 


\section{Ecuación 7}

$$
\text { Area Prom. }=\frac{\left(\text { Area Construida }{ }^{*} \text { No.Pisos }\right)}{(\text { No.Apartamentos })}
$$

- Área construida - obtenida del modelo.

- No. Pisos - obtenido del muestreo.

- No. Apartamentos - obtenido del muestreo.

Se presentan los resultados de la estimación del número de unidades residenciales, siguiendo el parámetro definido en esta sección para los edificios presentados. Posterior a esto, se realizó la prueba estadística $x^{2}$, a fin de evaluar si los resultados siguen una distribución normal.

Tabla 8. Resultados de la estimación del número de unidades residenciales

\begin{tabular}{|l|c|c|c|c|}
\hline \multicolumn{1}{|c|}{ ID Edificio } & Apartamentos & Año Const. & DHA,YoC & $\varepsilon$ HA,YoC \\
\hline $\begin{array}{l}\text { BLDG } \\
0003000000067203\end{array}$ & 36 & 1961 & 54 & -18 \\
\hline $\begin{array}{l}\text { BLDG } \\
0003000000069010\end{array}$ & 12 & 1899 & 21 & -9 \\
\hline $\begin{array}{l}\text { BLDG } \\
\text { 000300000006f0e2 }\end{array}$ & 12 & 1899 & 24 & -12 \\
\hline
\end{tabular}

Fuente. Elaboración propia del autor.

Los resultados del estadístico $\mathrm{x}^{2}$ arrojan lo siguiente:

$$
\begin{aligned}
& \sigma_{\mathrm{DHA}, \mathrm{YOC}}=7.367 \\
& D_{\mathrm{HA}, \mathrm{YOC}}: 356.6528>171.907
\end{aligned}
$$

Los resultados indican que el conjunto de datos no sigue una distribución, puede ser porque no representan de forma correcta el área de estudio y no son significantes, concluyendo que un nuevo muestreo debe realizarse. Esto puede deberse a diversos factores, uno puede ser la determinación del espacio habitable y el área construida del edificio. 
A pesar que el modelo de datos permite dividir el edificio en varias partes, esto no fue especificado tal y como lo expresa el estándar CityGML (Kolbe, Gröger, Nagel y Häfele, 2012), imposibilitando la identificación y diferenciación de las partes del edificio, por lo cual la representación gráfica no es leída de manera correcta. La (Figura 8a) muestra el modelo CityGML para un edificio dentro del área de estudio el cual presenta este problema. La línea blanca (Figura 8b) indica la forma como es leído el mismo edificio por la implementación, teniendo de fondo una imagen aérea de la zona.

El último factor es que no todos los edificios incluidos como residenciales están dedicados de forma exclusiva a este fin sino mezclan el primer o segundo piso a otros usos disminuyendo por consiguiente el número de apartamentos en el edificio.

Figura 8. Comparación de un edificio con respecto a su modelo según el desarrollo hecho

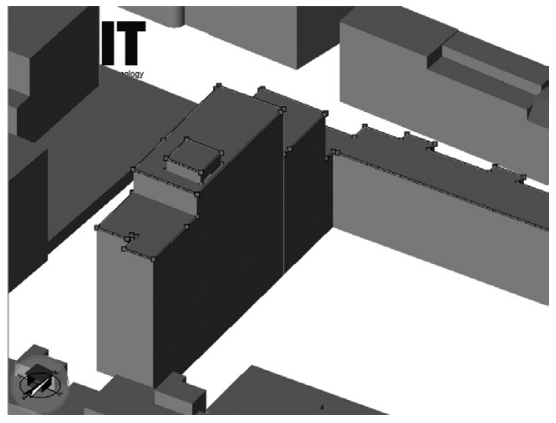

(a)

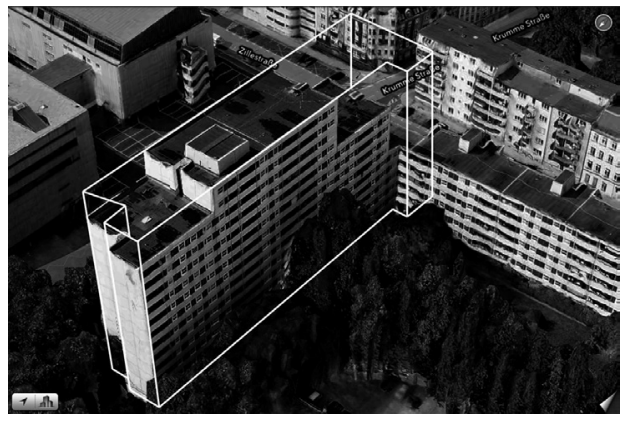

(b)

Fuente. Elaboración propia del autor.

\subsubsection{Número de habitantes por unidad residencial}

Para la estimación se utilizaron dos métodos, el primero, fue dividir el área promedio por apartamento, por el área por persona, multiplicando el valor entero de este resultado por el número de apartamentos del edificio. El segundo método divide el área neta construida del edificio, por el área por persona, luego se estima el número de personas por edificio y finalmente, se divide este valor por el número de apartamentos del mismo. 
Tabla 9. Número de habitantes por edificio

\begin{tabular}{|l|c|c|c|c|c|c|c|c|}
\hline & \multicolumn{3}{|c|}{ Método II } & \multicolumn{5}{c|}{ Método II } \\
\hline ID Edificio & Nh & $\begin{array}{c}\text { No. } \\
\text { Aptos }\end{array}$ & Nh,E & Nh,P & Nh,E & Nh & $\Delta h, \mathrm{~A}$ & $\Delta \mathrm{h}, \mathrm{E}$ \\
\hline $\begin{array}{l}\text { BLDG } \\
\text { 0003000000067203 }\end{array}$ & 3 & 63 & 189 & 11 & 99 & 2 & 1 & 90 \\
\hline $\begin{array}{l}\text { BLDG } \\
\text { 0003000000069010 }\end{array}$ & 4 & 21 & 84 & 6 & 42 & 2 & 2 & 42 \\
\hline $\begin{array}{l}\text { BLDG } \\
\text { 000300000006f0e2 }\end{array}$ & 5 & 24 & 120 & 9 & 54 & 3 & 2 & 66 \\
\hline
\end{tabular}

Fuente. Elaboración propia del autor.

Donde:

Nh - Número de habitantes por apartamento.

No. Aptos - Número de apartamentos en el edificio.

Nh,E - Número de habitantes por edificio.

Nh,P - Número de habitantes por piso.

$\Delta \mathrm{h}, \mathrm{A} \quad$ - Diferencia entre ambos métodos en el número de habitantes por apartamento.

$\Delta \mathrm{h}, \mathrm{E} \quad$ - Diferencia entre ambos métodos en el número de habitantes por edificio.

A fin de verificar la calidad de los resultados, estos se compararon con el promedio de habitantes por apartamento del área de estudio (1,64 habitantes) según los resultados del micro censo para el Estado de Berlín, 2011 (Amt für Statistik Berlin-Brandenburg, 2012).

Tabla 10. Comparación de los resultados de los dos métodos con respecto al promedio de habitantes por apartamento del área de estudio

\begin{tabular}{|l|c|c|c|c|c|c|}
\hline \multicolumn{1}{|c|}{ ID Edificio } & $\begin{array}{c}\text { No. } \\
\text { Aptos }\end{array}$ & $\begin{array}{c}\text { Prom. } \\
\text { Hab. }\end{array}$ & Mtd. I & $\Delta$ Mtd. I & Mtd. II & $\Delta$ Mtd. II \\
\hline $\begin{array}{l}\text { BLDG } \\
0003000000067203\end{array}$ & 63 & 103.32 & 189 & -86 & 99 & 4 \\
\hline $\begin{array}{l}\text { BLDG } \\
0003000000069010\end{array}$ & 21 & 34.44 & 84 & -50 & 42 & -8 \\
\hline $\begin{array}{l}\text { BLDG } \\
\text { 000300000006f0e2 }\end{array}$ & 24 & 39.36 & 120 & -81 & 54 & -15 \\
\hline
\end{tabular}

Fuente. Elaboración propia del autor. 
Prom. Hab: es el número de habitantes según el promedio por apartamento del área de estudio. La tabla 10 indica que los mejores resultados se obtienen a partir del segundo método.

\subsection{Consumo de energía eléctrica}

La estimación del consumo de energía eléctrica se hizo con base al número de unidades residenciales del edificio, número de habitantes por unidad residencial, número de aplicaciones energéticas que se asumieron como presentes en el edificio, así como el estilo de vida propuesto.

Inicialmente, se estimó el valor de consumo diario y se calculóo el semanal (cinco días laborales y dos festivos). El valor semanal es el marco de tiempo con el cual se estima el valor anual (un año tiene 52 semanas). Se presentan los resultados del consumo de energía por edificio, en donde es posible identificar que el área tiene un impacto directo en la estimación ya que para la metodología empleada en esta investigación, todos los parámetros exceptuando las aplicaciones energéticas están directamente relacionados con este valorm(Tabla 11).

Tabla 11.Resultados del consumo de energía (kWh / Año) por edificio para los diferentes estilos de vida

\begin{tabular}{|l|c|c|c|c|c|}
\hline \multicolumn{1}{|c|}{ ID Edificio } & $\begin{array}{c}\text { Área } \\
\text { Const. }\end{array}$ & $\begin{array}{c}\text { Alto } \\
\mathbf{1 0 0} \%\end{array}$ & $\begin{array}{c}\text { Medio } \\
\mathbf{5 0} \%\end{array}$ & Bajo $\mathbf{2 5 \%}$ & $\mathbf{1 5 \%}$ \\
\hline $\begin{array}{l}\text { BLDG } \\
\text { 00030000000667203 }\end{array}$ & 3589 & 454085.39 & 231668.91 & 120460.67 & 75977.37 \\
\hline $\begin{array}{l}\text { BLDG } \\
\text { 0003000000069010 }\end{array}$ & 1541 & 295191.75 & 149802.16 & 77107.37 & 48029.45 \\
\hline $\begin{array}{l}\text { BLDG } \\
\text { 000300000006e689 }\end{array}$ & 2331 & 421702.51 & 214003.09 & 110153.38 & 68613.51 \\
\hline
\end{tabular}

Fuente. Elaboración propia del autor.

Los resultados son lógicos partiendo del hecho que mientras más grande sea el edificio este requerirá una mayor demanda de energía, sin embargo, es importante tener presente que errores en la estimación de los parámetros, conllevará a una estimación errónea del consumo de energía. 
En el caso del consumo de energía por unidad residencial, los valores tienden a ser homogéneos lo cual se debe a que se asumió un estilo de vida estándar para todos los apartamentos, indicando que la variación del consumo sólo se basa en el aumento de su número de residentes.

Tabla 12. Consumos de energía (kWh / Año) por apartamento para los diferentes estilos de energía

\begin{tabular}{|l|c|c|c|c|c|}
\hline \multicolumn{1}{|c|}{ ID Edificio } & $\begin{array}{c}\text { No. } \\
\text { Apto. }\end{array}$ & $\begin{array}{c}\text { Alto } \\
\mathbf{1 0 0} \%\end{array}$ & $\begin{array}{c}\text { Medio } \\
\mathbf{5 0} \%\end{array}$ & $\begin{array}{c}\text { Bajo } \\
\mathbf{2 5 \%}\end{array}$ & $\mathbf{1 5 \%}$ \\
\hline $\begin{array}{l}\text { BLDG } \\
\text { 0003000000067203 }\end{array}$ & 54 & 8408.99 & 4290.16 & 2230.75 & 1406.99 \\
\hline $\begin{array}{l}\text { BLDG } \\
\text { 0003000000069010 }\end{array}$ & 21 & 14056.75 & 7133.43 & 3671.78 & 2287.12 \\
\hline $\begin{array}{l}\text { BLDG } \\
\text { 000300000006e689 }\end{array}$ & 30 & 14056.75 & 7133.43 & 3671.78 & 2287.12 \\
\hline
\end{tabular}

Fuente.Elaboración propia del autor. 


\section{Conclusiones}

La investigación presenta una propuesta para la estimación del consumo de energía de un edificio, usando un modelo 3D, sin embargo existen ciertos temas que deben discutirse.

Un modelo CityGML LOD2, es suficiente herramienta para la extracción de los parámetros del edificio que se mencionan en este documento y que son requeridos (unidades residenciales / habitantes). Sin embargo, existió un vacío en el conjunto de datos disponible para la fase de implementación que influenciaron en todo el proceso (faltaron el año de construcción, número de pisos, altura por piso, entre otros).

Una manera de solucionar estos problemas es emplear un modelo más detallado (LOD3 o LOD4); este último, es un modelo muy demandante y costoso de elaborar, aún más para una ciudad completa o una región.

Se puede hacer una observación al conjunto de datos usado (CityGML versión 1.0) referente a los techos. Esto se debe a que existen construcciones adicionales que no deberían estar conectadas con el edificio en si, sino ser parte del techo como un todo. 


\section{Referencias bibliográficas}

Amt für Statistik Berlin-Brandenburg. (Julio de 2011). Statistischer Recuperado de: http://www.statistik-berlin-brandenburg.de/Publikationen/Stat_Berichte/2011/SB_F1-1_01-10_BE.pdf

. (2012). Statistischer Bericht. Ergebnisse des Mikrozensus im Land Berlin 2011. Haushalte, Familien und Lebensformen. Amt für Statistik Berlin-Brandenburg.

Bayerische Staatregierung. (Agosto de 2007). Bayerische Bauordnung. Recuperado de: http://www.gesetze-bayern.de/jportal/portal/page/bsbayprod.psml?showdoccase=1|\&doc.id=jlr-BauOBY2007rahmenl\&doc.part=X

Bluejay, M. (Septiembre de 2010). How much electricity does my television use?. Recuperado de: http://michaelbluejay.com/electricity/ tv.html\#calculator

Branco, G., Lachal, B., Gallinelli, P., y Weber, W. (2004). Predicted versus observed heat consumption of a low energy multifamily complex in Switzerland based on long-term experimental data. Energy and Buildings, 36(6), 543 - 555.

Carbon Footprint Ltd. (s.f.). Household Energy Consumption. Recuperado el 5 de Marzo de 2013 de: http://www.carbonfootprint.com/energyconsumption.html

Carriòn, D. (2010). Estimation of the energetic rehabilitation state of buildings for the city of Berlin using a $3 D$ city model represented in CityGML. Berlin, Alemania: Technische Universität Berlin.

Clevenger, C. M., y Haymaker, J. (2006). The impact Of The Building Occupant On Energy Modeling Simulations. Stanford University. 
Coppock, J. T. y Rhind, D. W. (1991). Geographical Information Systems: Principles and Applications. En D. J. Manrique, M. F. Goodchild y D. W. Rhind (Edits.), The History of GIS (Vol. 1). Ottawa, Canada.

Cullen, K. A. (1999). Forecasting electricity demand using regression and Monte Carlo simulation under conditions of insufficient data. Morgantown, West Virginia. USA: West Virginia University Libraries. College of Agriculture, Forestry, and Consumer Sciences.

DestatisESTATIS. (Junio de 2009). Recuperado de: https://www.destatis.de/ DE/ZahlenFakten/GesellschaftStaat/EinkommenKonsumLebensbedingungen/Wohnen/Tabellen/HuG_Wohnflaeche_EVS.html

Eicker, U., Nouvel, R., Schulte, C., et al. (2012). 3D-Stadtmodelle für die Wärmebedarfberechnung. Fourth German-Austrian IBPSA Conference: BauSIM 2012. Berlin, Alemania.

FAO. (1991). Energy for Sustainable Rural Development Projects-1. training materials for agricultural planning. Rome, Italy: FAO.

Fujimoto, T., Shimoda, Y. y Yamaguchi, Y. (2011). Occupant Behaviour Model for Households to Estimate High-Temporal Resolution Residential Electricity Demand Profile. Proceedings of Building Simulation. Sydney, Australia.

Greenwood, P. E., y Nikulin, M. S. (1996). A Guide to Chi-Squared Testing. Wiley-Interscience.

Haldi, F. y Robinson, D. (2010). The Impact Of Occupants' Behaviour On Urban Energy Demand. BauSim 2010.

Hamburg Senat. (Diciembre, 2005). Hamburgische Bauordnung. Recuperado de: http://www.hamburg.de/contentblob/150654/data/hamburgische-bauordnung-hbauo).pdf

Ho, E. (Marzo, 2009). Edward Ho's Gadget Power Usage Page. Recuperado de: http://www.edho.com/power/ 
IGGS. (Abril de 2009). 3D Geo Database for CityGML (2.0.1 ed.). Berlin, Berlin, Alemania: Institute for Geodesy \& Geoinformation Science, Technische Universität Berlin.

IGGS. (Febrero de 2009). citygm/4j. Universität Berlin Institute for Geodesy \& Geoinformation Science and Technische. Recuperado de: http:// opportunity.bv.tu-berlin.de/software/projects/citygml4j/

KemaEMA, Inc. (2010). California Residential Appliance Saturation Study. Oakland, California: California Energy Commission.

KemaEMA-XenergyENERGY. (2004). California Statewide Residential Appliance Saturation Study. Oakland, California: California Energy Commission.

KIT. (Abril de 2012). FZKViewer. Karlsruhe Institute of Technology. Institute for Applied Computer Science Karlsruhe, Baden-Württemberg, Alemania. Recuperado de: http://www.citygmlwiki.org/index.php/ Freeware

Kolbe, T. H., Gröger, G., Nagel, C., et. al. (2012). OCG City Geography Markup Language (CityGML). Encoding Standard (Category: OpenGIS Implementation Specification ed.). Open Geospatial Consortium.

Lee, J. (2004). 3D GIS for Geo-coding Human Activity in Micro-scale Urban Environments. En Egenhofer, M. Freksa, C. y Miller H., Lecture Notes in Computer Science: Geographic Information Science 3234. 162178. Springer Berlin Heidelberg.

Lee, Y., Yi, Y., Malkawi, A. (2011). Simulating Human Behaviour and its Impact on Energy Uses. Proceedings of Building Simulation. Sydney, Australia, 1049-1056.

Loga, T., Diefenbach, D., Knissel, D., \& Born, R. (January de 2005). Entwicklung eines vereinfachten, statistisch abgesicherten Verfahrens zur Erhebung von Gebäudedaten für die Erstellung des Energieprofils von Gebäuden. Darmstadt: Institut Wohnen und Umwelt. 
Mehra, M. y Bharadwaj, A. (2000). Demand Forecasting for Electricity. En T. E. Institute (Ed.). Proceedings of the National Conference on Regulation in infrastructure Services: progress and way forward. New Dehli, India: The Energy and Resources Institute.

Meier, A., Rainer, L. y Greenberg, S. (1992). Miscellaneous Electrical Energy Use In Homes. Energy 17. 509-518.

Min.EnergiaMinas. (s.f.). Ministerio de Energía y Minas del Perú. Recuperado, de Guía del Consumo y Facturación de Energía Eléctrica.Recuperado de: http://intranet.minem.gob.pe/AppWeb/DGE/ CalculoConsumo

Mitchell, B., Ross, J., Park, R. et al. (1986). A Short Guide to Electric Utility Load Forecasting. Rand Corporation.

Nagel, C., Becker, T., Kaden, R. et al. (2010). Requirements and SpaceEvent Modelling for Indoor Navigation. Open Geospatial Consortium.

Neufert, E., Brockhaus, M., Kister, J. et al (2005). Bauentwurfslehre: Grundlagen, Normen, Vorschriften über Anlage, Bau, Gestaltung, Raumbedarf, Raumbeziehungen, Maße für Gebäude, Räume, Einrichtungen, Geräte mit dem Menschen als Maß und Ziel. Handbuch für den Baufachmann, Bauherr, Lehrenden und Lernenden (38 ed.). Alemania: Vieweg+Teubner Verlag.

Page, J., Robinson, D. y Scartezzini, J. L. (2007). Stochastic Simulation of Occupant Presence and Behaviour in Buildings. Building Simulation 2007. Beijing, China.

Phillips. (2013). Philips - Consumer Products. Recuperado de: http://www. philips.co.uk/c/

Princenton University. (2012). WordNet A lexical database for English. Electrical energy. Recuperado el 17 de April de 2013., de Electrical energy: Disponible en: http://wordnetweb.princeton.edu/perl/webwn?s=electrical\% 20energy 
Recht NRW. (Marzo 2000). Bauordnung für das Land Nordrhein-Westfalen. (M. f. Nordrhein-Westfalen, Editor). Recuperado de: https://recht.nrw. de/lmi/owa/pll_textl_anzeigen?vl_id=5820031106092333838

Reinhart, C. F. (2004). Lightswitch-2002: a model for manual and automated control of electric lighting and blinds. Solar Energy 77(1). 15 - 28.

Roetzel, A., Dietrich, U., Tsangrassoulis, A., y Busching, S. (Abril, 2010). Balancing buildings and occupants - a holistic approach to thermal comfort and greenhouse gas emissions in mixed mode offices. Adapting to Change: New Thinking on Comfort. Windsor Conference. England.

Santin, O. G., Itard, L., y Visscher, H. (2009). The effect of occupancy and building characteristics on energy use for space and water heating in Dutch residential stock. Energy and Buildings 41(11). 1223 - 1232.

Senatsverwaltung für Stadtentwicklung (2005). Entwurf ObjektschluüsseIkatalog Berlin (OSKA BIn) für die Automatisierte Liegenschaftskarte -ALK-, Alemania: Abteilung III - Geoinformation, Vermessung und Wertermittlung.

. (2010). Wohnenswerte Stadt / Wohnatlas Berlin. Einige Zahlen zum Wohnen in Berlin. Marktübersicht. Recuperado el 4 de Enero de 2010. , de Marktübersicht: Recuperado de: http:// www.stadtentwicklung.berlin.de/wohnen/wohnenswerte_stadt/de/ marktuebersicht.shtml

Senatsverwaltung für Stadtentwicklung und Umwelt. (2013). FIS Broker WMS. Gebäudealte 1992-1993.Recuperado de: http://fbinter.stadtberlin.de/fb/index.jsp

Shuvra, M., Rahman, M., Ali, A., y Khan, S. (2011). Modelling and Forecasting Demand for Electricity in Bangladesh: Econometrics Model. International Conference on Economics, Trade and Development IPEDR 7. Singapore. 
Sony. (2013). speaker. Recuperado de: http://www.sony.co.uk/product/mfiipod-speaker-docks/rdp-xa900ip/technical-specifications\#tab

Stratitum Energy. (2010). Cuánto consumen de electricidad los aparatos eléctricos.Recuperado de: http://www.gstriatum.com/energiasolar/articulosenergia/244-consumo-electricidad-aparatos-electricos.html

Wohnungsanwalt. (Junio, 2009). Wohnungen in Berlin. Recuperado de:http:// www.wohnungsanwalt.de/Wohnungsanwalt/Wohnungswirtschaft/ Statistik/Statistik/Wohnungen\%20Berlin.html

Wout, P., Dirk, S. y Hugo, H. (Mayo, 2010). Implementing realistic occupant behaviour in building energy simulations - the effect on the results of an optimisation of office buildings. Proceedings of the 10th REHVA World Congress: Sustainable Energy use in Buildings. 1 - 8. 